RESEARCH HIGHLIGHT

\title{
The role of memory in addiction: a commentary on Bornstein and Pickard memory sampling theory
}

\author{
Shiloh L. Echevarria-Cooper (iD) ${ }^{1}$ and Thorsten Kahnt (D) 1 ,2,3 \\ Neuropsychopharmacology (2020) 45:903-904; https://doi.org/10.1038/s41386-020-0627-x
}

Addiction is characterized by a failure to control drug use, despite negative consequences. Initial drug use is typically voluntary and driven by positive outcomes, such as high, euphoria, and social belonging. With repeated use, however, the decision to consume slips a person's voluntary control. This is evident in two key aspects of addiction. First, drug use continues in the face of negative consequences, such as negative impacts on health, financial instability, and eroding social relationships. Second, there is a high likelihood of relapse, that is, a sudden return to drug use even after prolonged abstinence.

Models of choice behavior, such as reinforcement learning (RL), have been proposed to account for the development of addiction. According to $\mathrm{RL}$, agents make choices that maximize reward, and they learn to expect rewards incrementally through experience. After each decision, the expected reward of the choice and its associated cues is updated based on the difference between the expected and received reward. In other words, what is learned and used to guide choices is a running average of the expected reward. In the case of addiction, a popular dogma is that initial positive experiences with the drug increase the expected reward of drug use and drug-related cues. Across time, its expected reward grows more powerful, resulting in compulsive drug use. In addition, with developing tolerance, drug use is further reinforced through negative reinforcement, namely, consumption to terminate aversive states of withdrawal [1]. Whereas early stages of drug use are well explained by $\mathrm{RL}$, the phenomena of continued drug use despite negative consequences and relapse after long periods of abstinence are not. With persistent use, the drug's positive effects diminish due to tolerance, and its negative effects may increase. $\mathrm{RL}$ predicts that when the drug provides less reward than predicted, the negative outcomes should be integrated into the running average, decreasing the expected reward of the drug and ultimately extinguishing use. This is not what happens; drug use typically continues, despite increasingly negative consequences. Moreover, RL predicts that successful abstinence indicates that an "unlearning" of drug-related cues and actions has occurred, and that drug use should become increasingly unlikely following longer periods of abstinence. Again, this is not what typically happens; relapse, even after prolonged abstinence, is a prominent feature of addiction. With standard RL accounts falling short in accounting for these two key phenomena of addiction, alternative models are needed. In this issue of Neuropsychopharmacology, Bornstein and Pickard propose such a model [2].

In the authors' memory sampling framework, a decision is guided by selective sampling of individual memories relevant to the decision, rather than by a running average of expected reward [3]. The value of a choice option is evaluated in the moment, based on a pseudo-random sample of memories of similar past choice outcomes. The choice that an individual makes in the present is thus largely driven by which past memories are being sampled. Memories with similar cues or context as the current situation are more likely to be sampled [4]. Bornstein \& Pickard suggest that memories of past positive drug experiences have a particularly high likelihood of being sampled, compared with more recent negative drug experiences. This is because initial drug experiences are highly salient, increasing their chance of being sampled. In addition, dopamine release induced by the drug may enhance the strength of the encoded memories making them more likely to be sampled. When sampled, these drugpositive memories bias the individual to choose the drug. The simple assumption that choices are driven by individual memories rather than by a running average helps to explain both continued drug use despite negative consequences and relapse after long periods of abstinence.

Bornstein and Pickard lay out how memory sampling is implicated in drug use across four stages of addiction: early use, persistent use, abstinence, and relapse. In each stage, the individual encounters drug-related cues. During early use, the cue becomes associated with positive drug experiences, and becomes relevant to a positive drug "fantasy", in which drug use is associated with positive effects, such as increased confidence, sociability, and euphoric feelings. As the individual devolves into persistent use, the encountered cues lead to the retrieval of memories regarding early, positive drug experiences, and the drug "fantasy". Over time, persistent drug use leads to increasingly negative consequences and diminishing rewarding outcomes, leading the individual to seek treatment and attempt abstinence. During treatment, medications may reduce the effects of drug craving and withdrawal, and cognitive therapies may encourage the individual to replace the drug "fantasy" with drug-inconsistent goals and associations. These may include improved relationships with family and friends, financial success, or improved health and longevity. At this stage, when the individual encounters drugrelated cues, they will retrieve early memories of positive drug experiences, but also retrieve memories regarding these druginconsistent goals and associations. This is how the memory sampling model can account for relapse after long periods of abstinence.

In contrast to the RL account, in which the expected reward of drug use should decrease in the face of negative experiences,

\footnotetext{
${ }^{1}$ Department of Neurology, Northwestern University Feinberg School of Medicine, Chicago, IL, USA; ${ }^{2}$ Department of Psychiatry and Behavioral Sciences, Northwestern University Feinberg School of Medicine, Chicago, IL, USA and ${ }^{3}$ Department of Psychology, Weinberg College of Arts and Sciences, Northwestern University, Evanston, IL, USA Correspondence: Thorsten Kahnt (thorsten.kahnt@northwestern.edu)
}

Received: 7 January 2020 Accepted: 10 January 2020

Published online: 31 January 2020 
positive drug memories in the sampling framework persist, and may, at any time, be sampled to reinstate drug use. In line with empirical evidence [5], the memory sampling model predicts that relapse is particularly likely in the presence of drug-related cues, single drug use, stress, or when drug-inconsistent goals seem out of reach. This is because all these situations enhance the likelihood that drug-positive memories are recalled over drug-inconsistent memories, either by directly strengthening positive drug memories, by weakening positive, drug-inconsistent memories, or by interfering with an individual's ability to effectively suppress drugpositive memories. With drug-positive memories preferentially sampled, the individual is more likely to consume the drug, and relapse.

The memory sampling framework proposed by Bornstein and Pickard does not only account for situations where the RL account fails. It also makes predictions about which mechanisms should be relevant for treatment success. For instance, cognitive therapy should encourage avoiding drug-positive cues and contexts where possible, alongside creating and strengthening positive, drug-inconsistent memories. Practicing recall of drug-inconsistent memories during therapy sessions may increase their likelihood of being sampled over drug-positive memories. Recall of druginconsistent memories may be further facilitated by associating them with personal reminders, such as photos, mementos, or smells. These reminders may then be used in situations previously associated with drug use to increase the likelihood of druginconsistent memories being sampled and outweighing memories associated with the drug "fantasy".

In conclusion, Bornstein and Pickard describe a novel framework that explains the early stages of drug use, as well as continued use in the face of negative consequences and relapse. Understanding addiction through the lens of memory sampling may inspire novel therapeutic approaches aimed at biasing memory sampling toward positive drug-inconsistent memories.

\section{FUNDING AND DISCLOSURE}

This work was supported by grants R01DC015426, R21DK118503, and T32AG020506. The authors declare that there are no competing financial interests.

\section{ACKNOWLEDGEMENTS}

We thank Dr. Yavin Shaham for insightful comments on this paper.

\section{AUTHOR CONTRIBUTIONS \\ S.L.E-C. and T.K. wrote the paper.}

\section{ADDITIONAL INFORMATION}

Publisher's note Springer Nature remains neutral with regard to jurisdictional claims in published maps and institutional affiliations.

\section{REFERENCES}

1. Wise RA, Koob GF. Neuropsychopharmacology. 2014;39:254-62.

2. Bornstein AM, Pickard H. Neuropsychopharmacology. 2020. https://doi.org/ 10.1038/s41386-019-0594-2.

3. Gershman SJ, Daw ND. Annu Rev Psychol. 2017;68:101-28.

4. Bornstein AM, Norman KA. Nat Neurosci. 2017;20:997-1003.

5. Bossert JM, Marchant NJ, Calu DJ, Shaham Y. Psychopharmacology. 2013; 229:453-76. 